\title{
Catharsis-based Counseling and Relaxation Training as Intervention for Reducing the Symptoms of Undifferentiated Somatoform Disorder: A Case Study
}

\author{
Fath Fatheya ${ }^{a *}$ and S. S. Budi Hartono ${ }^{\mathrm{b}}$ \\ ${ }^{a}$ Clinical Adult Magister-Profession, Faculty of Psychology, Universitas Indonesia, Depok, \\ Indonesia; ${ }^{b}$ Clinical Psychology Department, Faculty of Psychology, Universitas Indonesia, \\ Depok, Indonesia.
}

*Corresponding Author:

Fath Fatheya

Clinical Psychology Department

Faculty of Psychology, Universitas Indonesia

J1. Lkr. Kampus Raya, Depok, Jawa Barat

Indonesia, 16424

Tel.: +62 217270004

E-mail address: fatheya.fath@gmail.com 


\section{Catharsis-based Counseling and Relaxation Training as Intervention for Reducing the Symptoms of Undifferentiated Somatoform Disorder: A Case Study}

Somatoform disorder is a mental illness characterized by medically unexplained physical symptoms. The diagnosis of undifferentiated somatoform disorder is based on the occurrence of one or more physical complaints that persist for at least 6 months and that cannot be fully attributed to a medical condition. These complaints cause disproportionate levels of distress in the patient by inflicting longterm physical pain that cannot be alleviated through medical approaches. Many patients repeatedly change physicians to obtain a satisfying diagnosis only find that their symptoms cannot be fully attributed to a general medical condition. The psychological approach has a salient role in the treatment of somatoform disorder. Catharsis-based counseling therapy with relaxation training was originally designed to reduce the physical symptoms of undifferentiated somatoform disorder. This intervention was provided to a 26 -year-old woman presenting the physical complaints of migraine, genital itching, back pain, abdominal pain, and arm pain. The subject was experiencing emotional distress related to family issues. Through a pre-post test assessment approach, this study evaluated a series of interventions for reducing the intensity of physical complaints associated with undifferentiated somatoform disorders. Interventions were conducted in five sessions (one pre-assessment session, one catharsis-based counseling session, and three relaxation training sessions). The Screening for Somatoform Symptoms-7 (SOMS-7) tool was used to measure the intensity of physical complaints before and after intervention and at 3 months follow-up. SOMS-7 scores obtained before and after intervention revealed that the intensity of physical complaints perceived by the participant decreased after receiving catharsis-based counseling therapy with relaxation training. In conclusion, catharsis-based counseling and relaxation training decrease the intensity of physical complaints associated with undifferentiated somatoform disorders in emotionally distressed adults.

Keyword: catharsis; emotional distress; relaxation training; somatoform disorder; undifferentiated somatoform.

\section{Introduction}

Approximately $10 \%$ of the general population experience physical complaints without clear medical explanations (Rief \& Hiller, 2003). These complaints are excessive and cause disproportionate levels of distress by inflicting long-term and persistent pain. The term "somatoform disorders" was first introduced in the Third Edition of Diagnostic and Statistical Manual as an acknowledgment that numerous individuals experience somatic distress that do not fit the criteria of physical diseases, anxiety, mood, or psychotic disorders (Rief \& Hiller, 2003). Hiller et al. (2006) suggested that the term "somatoform" is a clinical condition without a pathological origin despite giving the impression of somatic disease. Rief et al. (2001) stated that the most common somatoform symptoms are back pain, joint pain, headache, abdominal 
symptoms, and cardiovascular symptoms. The main characteristics of undifferentiated somatoform disorder include one or more physical complaints without medical explanation. These complaints may persist for at least 6 months and cause clinically significant distress or impair daily activities. The prevalence of undifferentiated somatoform disorder is high and ranges from $10 \%-30 \%$ of the population of the UK, Norway, and China. Nevertheless, studies focusing on undifferentiated somatoform disorder are relatively scarce (Koh, 2013).

Kellner (1994) found that the somatoform symptoms of certain patients have an etiological basis in psychological processes. Although somatization symptoms often have a psychological, rather than physical, origin, these symptoms do have a physiological basis (Sharpe \& Bass, 1992). Physical complaints arise from complex interactions among biological, psychological, and social processes. Rief and Hiller (2009) assumed that psychological factors play a crucial role in the treatment of somatoform disorders. Therefore, psychological intervention is important for the management of somatoform disorders. Traumatic experiences, negative affectivity, insecure attachment style (fearful), and high emotional arousal levels are some risk factors of somatic complaints (Rief \& Boradbent, 2007; Witthoft, Basfeld, \& Steinhoff, 2012). Emotion regulation skill is also a factor in somatic complaints that is often addressed, especially the "anger-in" style. Pain usually increases the intensity of negative emotion, and conversely, negative emotion increases the intensity of pain and induces further somatosensory experiences. Thus, the development of somatoform disorders is a vicious cycle. A client who fears rejection and believes that they will be neglected demonstrates an insecure attachment style in their interpersonal interactions.

Somatoform disorders cause considerable disability among individuals, and their treatment increases health-care costs (Allen, Woolfold, Escobar, Gara, \& Hamer, 2006; Barsky, Orav, \& Bates, 2005). Clients with somatoform disorders exhibit irrefutable symptoms of physical complaints although the causes of their complaints lack medical evidence (Sharpee \& Bass, 1992). Somatoform disorders are often resistant to therapy and are inexplicable because the use of medical drugs is often insufficient for mitigating symptoms. Individuals with somatoform disorders frequently and persistently seek medical treatment and undergo expensive diagnostic procedures.

Many studies have revealed the high comorbidity between depression and somatofrom disorders (Hiller, Rief, Fichter, 1995; Rief, Nanke, Emmerich, Bender, \& Zech, 2004; Rief, Hennings, Riemer, \& Euteneur, 2010). Comorbid somatoform disorder, depression, and anxiety have a psychological influence as opposed somatoform disorder without depression (Rief et al., 2004). The somatic symptoms of depression are associated with anxiety, tendency to amplify somatic distress, and difficulty in communicating emotional distress (Sayar, Kirmayer, \& Taillefer, 2003). Acute anxiety, somatosensory amplification, and alexithymia are some predictors of the somatic symptoms of depressive disorders. Sharpe and Bass (1992) established a model suggesting that anxiety is a psychological aspect of the development of somatoform disorders. Anxiety is caused by life stresses in a social aspect, and its existence causes physical symptoms, such as muscle tension, hyperventilation, and autonomic activity. These effects turn into somatic sensation, which is then interpreted as physical illness. Perceived ill health induces stress, which increases anxiety in individuals. Similarly, undifferentiated somatoform disorders have a high psychological influence. Johnson et al. (1981) found that most individuals with physical complaints have experienced emotional or family issues. 
Kirmayer et al. (1994) showed that personality traits contribute to somatoform disorders. Negative affectivity (neuroticism) is associated with high levels of somatic and emotional distress. Individuals with high negative affectivity have a physiological tendency to experience a broad range of somatic symptoms. Introversion is also associated with increased physiological responses to sensory stimuli, including pain. A high level of muscular tension may also cause local aches and pains. Sainsbury and Gibson (in Sharpe \& Bass, 1992) used electromyograms to identify associations between the sites of bodily symptoms and elevated muscle tension.

Psychological intervention plays an important role in relieving somatoform symptoms. The cognitive behavioral therapy (CBT) approach is the most widely studied approach for the treatment of somatoform disorders. CBT is effective in overcoming somatization symptoms (Allen et al., 2006; Kroenke \& Swindle, 2000; Rief, 2010). An empirical evaluation of studies using CBT to deal with somatoform disorder suggested that relaxation training is often included in the CBT series (Woolfolk \& Allen, 2012). Allen et al. (2006) studied the provision of CBT to individuals with somatoform disorders and who received relaxation technique training in their therapeutic sequences for reducing physiological arousal.

In Germany, Austria, and Switzerland, relaxation is a widely used somatopsychoterapeutic intervention in the treatment of psychosomatic disorders (Lahmann et al., 2009). The therapeutic effect of relaxation is obtained through the positive stimulation of the autonomic nervous system. Research conducted by Lahmann et al. (2009) proved the application of relaxation can reduce asthma in patients for up to 4 months of after the intervention. Schröder et al. (2013) examined the application of CBT programs and relaxation training (progressive muscle) to overcome somatoform symptoms. Randomization indicated that the effects obtained through the implementation of the CBT program and relaxation training are not significantly different. Both methods are equally effective in reducing the number and intensity of somatoform symptoms at 6 months follow-up. Moreover, approaches that promote emotional regulation and stress reduction have to be integrated into current psychological treatments for somatoform disorders (Koh, 2013).

Treatments based on cathartic methods were first introduced by Freud and Breuer (Nichols \& Efran, 1985) who initially handled cases of hysteria with symptoms of muscle contractions, seizures, and numbness of various parts of the body. At that time, they discovered that emotions inflicted by a certain traumatic memory from the past can permanently terminate the symptoms of hysteria in their client. The client is asked to explain the traumatic event in as much detail as possible and verbally convey their emotions toward the event. The catharsis approach in counseling requires the client to connect to their feelings and express emotions that are painful to face or difficult to contain (Jinks, 2012). Emotional problems are treated by encouraging the patient to express their repressed or restrained emotion. In other words, catharsis is a purgation of emotions.

Nichols and Efran (1985) attempted to analogize emotions as a system of hydraulics. For example sadness, is said to "pour up," anger "boils," happiness "bubbles over," and grief "comes in waves (cycle)." Emotions are considered as watery substances that flow toward the gate of conscious expression. Blocked emotions accumulate pressure and seek escape through another path. Therefore, emotions require a discharge. Guinagh (1987) stated that emotional expression is typically expressed through anger and misery. The cathartic experience itself holds the risk of 
losing control. Nevertheless, emotional expression will generally decrease in intensity. For example, prolonged sobbing eventually becomes quiet weeping. In this phase, emotional expression is consciously generated for therapeutic purpose. Cathartic individuals will thus feel less tense. Some scholars believe that catharsis is followed by decreased tension, increased mental clarity and feelings of well-being, and emotional stability (Guinagh, 1987; Scheff, 1979). The therapist determines whether their client needs this type of intervention. Providing a cathartic counseling approach is frequently necessary when individuals are reluctant to open up about their actual experiences for fear of their inability to handle the situation. The therapist needs to concentrate on responding empathetically and gradually guiding the client to deep contact with their emotions (Jinks, 2012).

Relaxation therapies may remove physical symptoms, reduce emotional suppression, improve quality of life, and increase well-being (Guinagh, 1987; Koh, 2013). Benson (as cited in Guinagh, 1987) stated that relaxation slows down physiological and behavioral states. Regularly activating this relaxation response can reduce physical and emotional complications. Relaxation therapies are easy to learn and can be practiced by patients on their own (Guinagh, 1987). Relaxation exercise helps relieve depression symptoms, anxiety, sleep disturbance, fatigue, pain, nausea, and vomiting. Relaxation therapy enables patients to exercise control over their own bodies and relieve pain and anxiety without medication. Furthermore, relaxation as a stressreducing intervention can improve immune function (Kiecolt-Glaser \& Glaser, 1986).

Johnson et al. (1981) studied the exploratory use of group relaxation training to attenuate various somatic complaints. In general, the somatic complaints of the clients reduced after six sessions. Lidbeck (1997) compared the effectiveness of CBT (dedicated to patient education and stress relaxation) in a group of patients with somatization disorder and in a control group comprising individuals on an 8-month waiting list. The somatic preoccupation and perception of illness of the first treatment group moderately improved. Approximately two-thirds of the patients claimed that relaxation training helped improve their subjective coping skills. The use of functional relaxation techniques combined with guided imagery has been provided (Lahmann, Loew, Tritt, dan Nickel, 2008; Lahmann, dkk., 2009) to clients with asthma and somatoform heart disorder (chest pain) and have yielded significant positive effects in both groups of participants.

This study aimed to review the evidence supporting the effectiveness of cathartic-based counseling and relaxation training in the treatment of emotionally distressed adults with undifferentiated somatoform disorders. This study is a single-subject case study and involved the psychological treatment of a client at a campus counseling center. The researcher, a graduate student of the psychology magister profession, conducted the intervention under the supervision of lecturers who are experts in the adult clinical field and who have been certified by Himpunan Psikologi Indonesia. The implementation of the intervention program presented in this study is expected to be replicated for further research or intervention in related disorders.

\section{Methods}

\section{Participant}

The subject of this study is a woman who works as a student administration staff at a public university. In this work, the subject is referred to with the pseudonym Zee. The subject registered on her own will with the campus counseling center to receive psychological treatment for her 
problems. She then then made an appointment facilitated by the counseling center to meet the researcher for psychological intervention. A semi-structured interview was conducted to assess the subject and to determine her life history and psychological problems.

\section{Case introduction}

Zee is a 26-year-old unmarried woman. She is the oldest of two siblings and currently lives at home with her mother and sister. Zee has finished her diploma-3 education and is currently working toward a bachelor's degree at an open university while working full time as faculty staff. Although she has long felt the need to receive professional help, she was only able to fully realize that she required professional help upon her first meeting with the researcher.

\section{Presenting complaints}

During her first meeting with the researcher, Zee cried while telling her story. Zee spoke about the separation of her parents in 2012 and their official divorce in 2014. Since her parents' divorce in 2014, Zee had suffered migraine attacks almost daily. Zee had consulted with a general practitioner regarding her migraines. A physical examination of her head revealed no discernible medical disorders. Zee was prescribed a painkiller, which she only took when her migraines are severe. However, she did not take the painkiller regularly because she believes that the constant consumption of painkillers may be harmful. In 2015, Zee first felt stiffness in her lower neck near the shoulder. The stiffness was extremely painful particularly upon waking up. She claimed that the stiffness caused severe discomfort that made her lose the will to get up and live the day. Zee had expressed her complaints to a neurologist, but no specific medical conditions were found to be their cause.

Zee had felt genital itching since April 2016. The itching was disturbing and would worsen by nighttime. Zee would often wake up at night because of the itching. Zee had her genitalia medically examined. However, they were physically healthy without any disorder. Zee was prescribed an ointment to reduce the itching. The ointment, however, does not always work. Doctors suspected that the itching was caused by distress or psychological burden. This opinion reinforced her desire to see a psychologist to confirm whether her discomfort stems from a psychological issue.

Since her childhood, Zee's parents would often quarrel. Zee saw her mother as a figure who would often act roughly. She would scream; curse; and vent her emotions on Zee, her father, or her sister. By contrast, Zee's father was submissive, always kept his voice down, and ignored the bad temper of his wife. This inharmonious relationship continued until Zee reached adulthood. Zee had been trying to repress her family problems and told no one about her family problems because she though that they were a disgrace not worthy of exposure to others. During adolescence, Zee had a boyfriend, and the presence of this boy had been very helpful in keeping her mind distracted from her family problems. In 2012, Zee broke up with her boyfriend of 6 years. The end of this relationship forced Zee to deal with her family problems. Since then, Zee had been exposed to the quarrels of her parents and had begun to ruminate deeply on the issue. Zee harbored sadness and resentment toward her mother's bad temper and felt sorry for her father's agonizing situation.

In 2014, Zee's parents legally divorced after 2 years of separation. Zee has been estranged from her mother since her parents' divorce and kept communications to a minimum until 2016. In 2015, Zee's father remarried a woman who later became Zee's stepmother. Zee felt an 
intrapsychic conflict where on the one hand, she was glad her father found a person who can take care of him. On the other hand, she deeply mourned her own parents' divorce. Until now, Zee's mother has not remarried and lives with Zee and her sister. Zee and her sister would meet their father once a month, though irregularly. Zee is financially self-sufficient and does not depend on her father but her father continues to financially provide for Zee's sister who is still in school. Zee considers her relationship with her father as well-established in contrast with her poor relationship with her mother.

Zee showed signs of depression during the psychological examination. She also cried during the first and second meetings with the researcher. At the beginning of the session, Zee would often lower her tone and volume and would lower her head while talking to the researcher. During the counseling session, Zee actively asked the researcher to help her understand her condition. She was quite responsive and able to derive good insights from her problems. In general, Zee engaged cooperatively and collaboratively during every session.

Table 1

Multiaxial Diagnosis for Zee

\begin{tabular}{ll}
\hline Axis I & 300.82 Undifferentiated Somatoform Disorder \\
Axis II & V71.09 No Diagnosis \\
Axis III & 558.9 Gastroenteritis \\
Axis IV & 61.20 Parent-child Relationship \\
Axis V & General Adaptive Functioning 61-70.
\end{tabular}

Note. Diagnosis based on Diagnostic and Statistical Manual of Mental Disorder, $4^{\text {th }}$ ed., text rev. Adapted from Diagnostic and Statistical Manual of Mental Disorder IV TR by the American Psychiatric Association, 2000, Washington, DC: American Psychiatric Association.

\section{Research Design}

This research used a single-case study design and a pre-post intervention evaluation design. The participant's somatoform symptoms were evaluated before the intervention phase (pre-test), after the intervention phase (post-test), and at the 3-month follow-up after the intervention. The series of studies occurred over a span of 14 days for five meetings, with sessions adjusted to the availability of the participant. The first session was conducted 4 days after the pre-session. The second to fourth sessions were conducted with intervals of 3, 2, and 1 days. Each session lasted for approximately 90 minutes.

Table 2

Intervention Design

\begin{tabular}{|c|c|c|}
\hline Sessions & Agenda & $\begin{array}{l}\text { Time Interval } \\
\text { (within } 14 \text { days) }\end{array}$ \\
\hline Pre-Session & Initial interview and assessment. & - \\
\hline Session 1 & Catharsis-based counseling and psychoeducation & 4 days \\
\hline Session 2 & Relaxation 1 (Progressive muscle relaxation) & 3 days \\
\hline Session 3 & $\begin{array}{l}\text { Relaxation } 2 \text { (Deep breathing, diaphragm breathing, and fingertip } \\
\text { sensing) }\end{array}$ & 2 days \\
\hline Session 4 & $\begin{array}{l}\text { Relaxation } 3 \text { (Grounding emotion stabilization and guided imagery) } \\
\text { and termination. }\end{array}$ & 1 day \\
\hline
\end{tabular}




\section{Measures}

The intervention was provided to reduce the symptoms of somatoform disorder in the participant. Somatoform symptoms were measured using Screening for Somatoform Symptoms-7 (SOMS-7) by Rief and Hiller (2003). SOMS-7 uses a Likert 0-4 scale ranging from "not at all" to "very severe" to represent the frequency of somatoform symptoms within the last 7 days. The results of pre-test, post-test, and follow-up SOMS-7 were then compared to determine changes in the intensity and frequency of somatoform symptoms.

\section{Procedure}

The entire process of the intervention was conducted in the counseling room at the campus counseling center. The researcher (the first author) conducted the entire procedure of the study with the participant under the supervision of the second author. The series of interventions consisted of two main components, namely: (1) catharsis-based counseling and (2) relaxation training. The relaxation training program consisted of progressive muscle relaxation, deepbreathing technique, and guided imagery relaxation.

\section{Catharsis-based Counseling}

Catharsis-based counseling was given to Zee during the first session after the initial interview. Catharsis-based counseling is advised to last for long durations, such as 90 min (Jinks, 2012). After the first meeting, the researcher conducted the assessment and saw the need for the participant to experience catharsis to reduce emotional distress. At the beginning of each subsequent session, the researcher provided counseling through the catharsis approach to the participant. The researcher let the course of the session be determined by the participant and was not directive, only asking open-ended questions to help the participant connect with her feelings toward past events. The researcher focused on the participant's feelings at the moment while interpreting past events instead of asking in detail about events. The researcher used active listening techniques and emphatic response to help the participant connect with her deep emotions. Staying focused on the client's emotions and responding accordingly while providing a safe environment for the disposal of the client's emotions are essential skills for managing catharsis-based counseling. Sharing what has been newly noticed by the participant will help the participant deeply examine their feelings. At the end of the session, the researcher would ask what the participant has discovered or learned during the session. During the end of the first session, the researcher also provided psychoeducation about somatoform disorder and the importance of emotional processing for maintaining mind-body balance.

\section{Relaxation Training}

Relaxation training through progressive muscle relaxation was introduced during the second session. Given that individuals with somatoform disorder are sensitive to bodily sensation, the researcher suggested that progressive muscle relaxation would help the participant experience the difference between tension and relaxation. Relaxation training should be learned early on during the intervention to provide a basis for the participant to know how their body would feel when relaxed. The researcher asked Zee to sit in a comfortable and quiet position on a chair. In general, Zee could follow most of the given instructions, but Zee had difficulty following instructions on relaxing the muscles of her eyes, mouth, and heels. The researcher then help Zee with instructions that she found difficult. 
By the third session, the researcher provided relaxation training with deep-breathing techniques. The deep-breathing exercise began by counting the number of reflex breaths for $1 \mathrm{~min}$, followed by counting the number of deep breaths that the participant could draw in $1 \mathrm{~min}$. Under normal circumstances, Zee could breathe 15 times in $1 \mathrm{~min}$. The researcher then asked Zee to take the deepest breaths she could, and Zee scored 7 deep breaths per min. When asked to breathe normally again, Zee's breathing decreased from 15 to 12 breaths per min. Lastly, Zee was asked to take the deepest possible breaths for $1 \mathrm{~min}$, and she was able to deeply breathe 5 times per min. This training aims to enable participants to relax by regulating their respiratory patterns.

The researcher then taught the participant to breathe using her diaphragm and explained the difference between breathing using the lungs and diaphragm. Zee was asked to observe how she would breathe normally using the lungs. The researcher demonstrated how to control breathing by using the abdominal muscles. Zee was guided to perform diaphragmatic breathing. Zee had difficulty immediately understanding the difference between lung and diaphragmatic breathing. The researcher continued to monitor and guide Zee until she was able to master diaphragmatic breathing.

Next, the researcher taught Zee the sensing-fingertip technique. Zee was directed to close her eyes and unite the tips of the fingers of both hands. The researcher instructed Zee to focus on her breathing and her fingertips and to feel the pulse on her fingertips. Zee initially had trouble focusing but the researcher gave her time and directed her until she was able to feel her pulse on at least three fingertips. After the third session ended, Zee admitted that she that felt her body become lighter and slightly drowsy. She felt relaxed. Zee was asked to practice the techniques she learned at home.

The fourth and final session began with grounding emotion stabilization technique. Zee was asked to lie down on the relaxation chair, breathe regularly, and focus on things that bring out neutral/positive emotions. Zee had difficultly identifying objects that induced neutral/positive emotions and instead provided objects that induced negative emotions. The researchers gave Zee time to calm down and instructed Zee to focus on her search for objects that induced neutral/positive emotions. Zee was able identify three objects that corresponded to neutral/positive emotions, namely, drinking glass, door key holes, and tables. The researcher expressed their appreciation and asked Zee to look for two more objects that provided neutral/ positive emotions. The researcher then directed Zee to close her eyes and use her sense of hearing to name at least three sounds that she heard. Afterward, the researcher asked Zee to focus her attention and mention three things that are related to the sense of touch.

For the closing session of the day, the researcher provided guided imagery relaxation. Zee was asked to sit in a comfortable position and begin by taking a deep breath. The researcher then slowly read the guided imagery script and asked Zee to focus on herself. Guided imagery relaxation aims to increase tolerance and acceptance of bodily sensation. Zee could follow the researcher's instructions and visualize the instruction read by the researcher. After the session, Zee admitted that her body felt lighter, calmer, and more comfortable than before.

\section{Termination}

After the entire series of intervention was conducted, the researcher ended the session with termination. Termination included a review of what has been learned and new understandings of 
Zee's personal self and experience of undifferentiated somatoform disorder. The researcher also reviewed the provided relaxation techniques and encouraged Zee to practice the relaxation techniques. Post-test assessment was performed during the termination phase. Zee's somatoform symptoms were assessed using SOMS-7. In general, Zee showed a positive attitude toward the intervention. During counseling, Zee stated that she felt increasingly relaxed.

\section{Data Analysis}

After the intervention ended, the researcher analyzed the participant's SOMS-7 data. Data were analyzed by creating a comparison chart of each somatic complaint perceived by Zee at pre- and post-intervention and at 3 month follow-up after the intervention.

\section{Results}

The intensity of Zee's somatoform symptoms reduced after intervention. At the beginning of the intervention, Zee would experience genital itching almost every night despite receiving treatment with a prescription ointment. After the intervention, Zee felt a decline in the disturbance caused by genital itching. During the intervention session, Zee even admitted to not remembering having experienced genital itching. By the evening after finishing the third session, Zee admitted to sleeping soundly, whereas 2 days prior to the intervention she felt unable to sleep because of the pain in her arms. The development of Zee's somatic complaints before and after undergoing intervention is presented in Fig. 1.

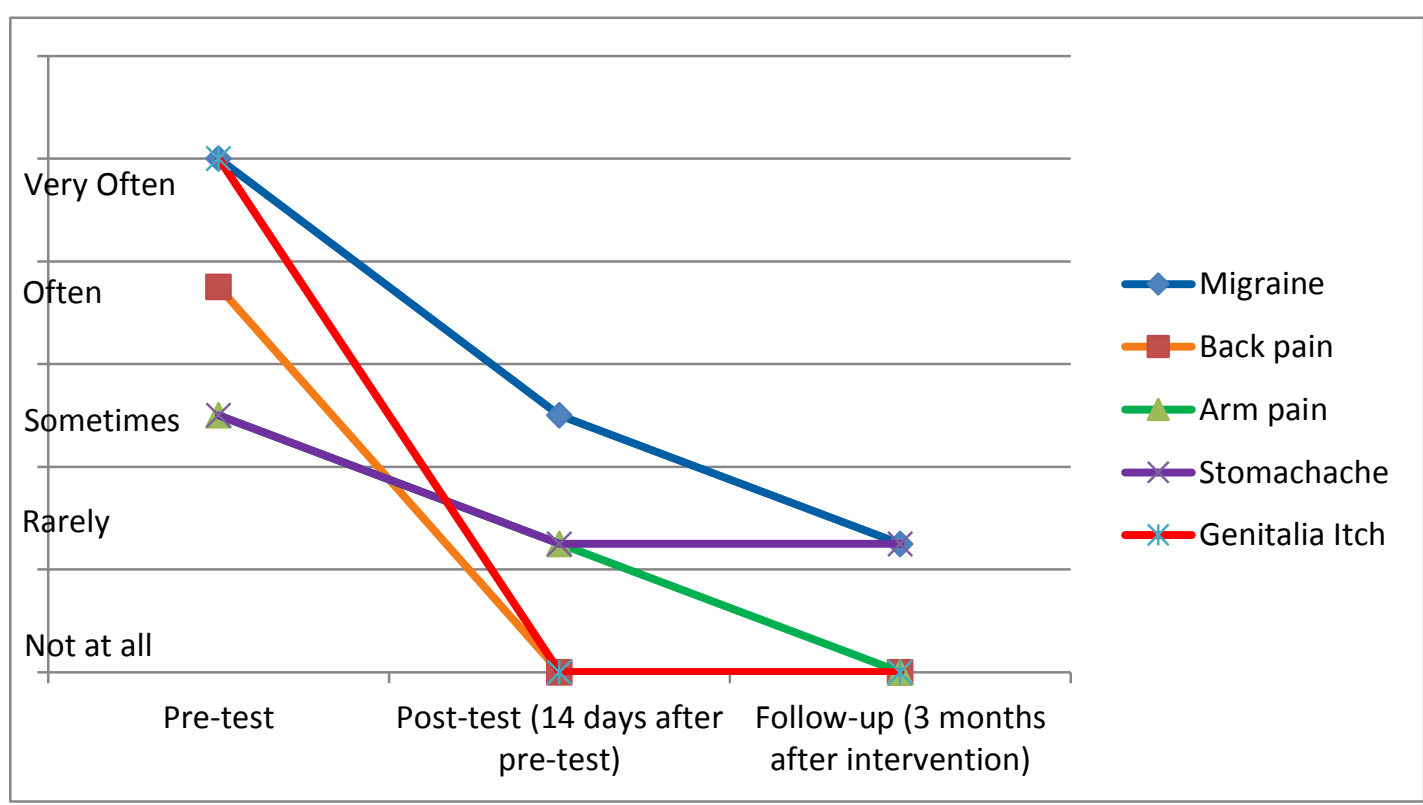

Figure 1. Development of Zee's somatic complaints. 


\section{Follow-up}

A follow up was conducted 3 months after the intervention. The researcher arranged another meeting with Zee to assess her condition. The intensity of Zee's physical complaints had remarkably reduced. Zee admitted that she no longer felt disturbed by somatic complaints and had stopped taking medication to overcome her somatic complaints. She would occasionally feel exhausted from work and her migraine would relapse. These symptoms, however, were not sufficiently severe to interfere with her daily life.

\section{Discussion}

In general, catharsis-based counseling and relaxation training promises to reduce the intensity of undifferentiated somatoform symptoms in an emotionally distressed adult. This study's participant (Zee) claimed to have gained benefits and exhibited progress after receiving intervention. Zee's willingness to open up and engage in the intervention process definitely influenced the outcome of the intervention, especially because she has a strong internal motivation to receive treatment and sufficient intellectual potential to understand the insights that she had gained. The positive outcome of this intervention also continued after 3 months postintervention. This result confirmed that the intervention can effectively maintain reduce Zee's somatoform symptoms over the long term.

This study has numerous limitations despite its positive outcomes. The context in which Zee has never expressed her emotions and talked about her family issues is very specific and thus cannot be generalized to other cases. Moreover, the time span of this intervention is relatively short. Additional time is necessary to monitor the participant's mastery of the provided relaxation techniques. The researcher also did not closely monitor how frequently the participant practiced the relaxation techniques at home. The SOMS-7 instrument is superficial and should be supported by other tools for measuring the frequency and duration of somatic complaints. Furthermore, future studies should measure and monitor the perceived changes in the level of emotional distress and whether such changes are directly correlated with physical symptoms. The present study can be extended using a large sample and comparison group to draw definitive conclusions regarding the effectiveness of the present intervention.

\section{References}

Allen, L. A., Woolfolk, R. L., Escobar, J. I., Gara, M. A., \& Hamer, R. M. (2006). Cognitive-behavioral therapy for somatization disorder: A randomized controlled trial. Archive of Internal Medicine, 166, 1512-1518.

American Psychiatric Association. (2000). Diagnostic and statistical manual of mental disorders: DSM-IV-TR. Washington, DC: American Psychiatric Association.

Barsky, A. J., Orav, E.., \& Bates, D. W. (2005). Somatization increases medical utilization and costs independent of psychiatric and medical comorbidity. Archives of General Psychiatry,62, 903-910.

Guinagh, B. (1987). Catharsis and cognition in psychotherapy. New York: Springer-Verlag.

Hiller, W., Rief, W., \& Brähler, E. (2006). Somatization in the population: From mild bodily misperceptions to disabling symptoms. Social Psychiatry and Psychiatric Epidemology,41, 704-712.

Jinks, G. (2012). Specific strategies and techniques. In C. Feltham \& I. Horton ( $3^{\text {rd }}$ ed.), The sage handbook of psychotherapy and coounselling (pp.87-108). London, UK: Sage.

Johnson, C., Shenoy, R. S., \& Langer, S. (1981). Relaxation therapy for somatoform disorders. Hospital and Community Psychiatry,32(6), 423-424.

Koh, K. B. (2013). Somatization and psychosomatic sypmtoms. New York: Springer. DOI: 10.1007/978-1-4614-7119-6.

Kellner, R. (1994). Psychosomatic syndromes, somatization and somatoform disorders. Psychotherapy and Psychosomatics,61, 4-24. 
Kiecolt-Glaser, J. K., \& Glaser, R. (1986). Psychological influences on immunity. Psychosomatics,27(9), 6221-624.

Kirmayer, L. J., Robbins, J. M., \& Paris, J. (1994). Somatoform disorders: Personality and the social matrix of somatic distress. Journal of Abnormal Psychology, 103(1), 125-136.

Kroenke, K., \& Swindle., R. (2000). Cognitive-behavioral tehrapy for somatization and symptom syndromes: A critical review of controlled clinical trials. Psychotherapy and Psychosomatics, 69, 205-215.

Lahmann, C., Loew, T. H., Tritt, K., \& Nickel, M. (2008). Efficacy of functional relaxation and patient education in the treatment of somatoform deart disorders: A randomized, controlled clinical investigation. Psychosomatic, 49(5), 378-385.

Lahmann, C., Nickel, M., Schuster, T., Sauer, N., Ronel, J., Noll-Hussong, M., Tritt, K., Nowak, D., Rohricht, F., Loew, T. (2009). Functional relaxation and guided imagery as complementary therapy in asthma: A randomized controlled clinical trial. Psychotherapy and Psychosomatics, 78, 233-239.

Lidbeck, J. (1997). Group therapy for somatization disorders in general prpactice: Effectiveness of a short cognitive-behavioral treatment model. Acta Psychiatrica Scandinavica,96, 14-24.

Nichols, M. P., \& Efran, J. S. (1985). Catharsis in psychotherapy: A new perspective. Psychotherapy,22(1), 46-58.

Rief, W. (2010). Painting the picture of distressing somatic symptoms. Journal of Psychosomatic Research,68, 1-3.

Rief, W., Nanke, A., Emmerich, J., Bender, A., \& Zech, T. (2004). Causal illness attributions in somatoform disorders: Associations with comorbidity and illness behavior. Journal of Psychosomatic Research,57. 367-371.

Rief, W., Hessel, A., \& Braehler, E. (2001). Somatization symptoms and hypochondriacal features in the general population. Psychosomatic Medicine, 63, 585-602.

Rief, W., \& Hiller, W. (2003). A new approcah to the assessment of the treatment effects of somatoform disorders. Psychosomatic,44(6). 492-298.

Rief, W., Hiller, W., \& Fichter, M. M. (1995). Somatoform symptoms in depressive and panic syndromes. International Journal of Behavioral Medicine,2(1), 51-65.

Rief, W., Hennings, A., Riemer, S., \& Euteneur, F. (2010). Psychobiological differences between depression and somatization. Journal of Psychosomatic Research,68,495-502.

Rief, W., \& Boradbent, E. (2007). Explaining medically unexplained symptoms-models and mechanisms. Clinical Psychology Review, 28, 821-841.

Sayar, K., Kirmayer, L. J., \& Taillefer, S. S. (2003). Predictors of somatic symptoms in depressive disorder. General Hospital Psychiatry, 25, 108-114.

Sharpe, M., \& Bass, C. (1992). Pathophysiological mechanisms in somatization. International Review of Psychiatry,4(1),81-97. DOI:10.3109/09540269209066305

Schröder, A., Heider, J., Zaby, A., \& Göllner, R. (2013). Cognitive behavioral therapy versus progressive muscle relaxation training for multiple somatoform symptoms: Results of a randomized controlled trial. Cognitive Therapy and Research,37,296-306.

Witthoft, R. L., Basfeld, C., Steinhoff, M., \& Gerlach, A. L. (2012). Can't suppress this feeling: Automatic negative evaluations of somatosensory stimuly are related to the experience of somatic symptom distress. Emotion, 12(3), 640-649.

Woolfolk, R. L., \& Allen, L. A. (2012). Cognitive behavioral therapy for somatoform disorders. In Irismar R. I., Standard and innovative strategies in cognitive behavior therapy (pp. 118-144). Rijeka: InTech. 